\title{
Wie authentisch führe ich? Prüfung der Selbsteinschätzungsform des Deutschen Inventars Authentischer Führung
}

\author{
Magdalena Reineboth ${ }^{1}$ Nensy Le Thu Ha ${ }^{1} \cdot$ Luise Franke-Bartholdt $^{1} \cdot$ Dirk Frömmer $^{2} \cdot$ Jürgen Wegge $^{2}$ (D) . \\ Anja Strobel ${ }^{1}$
}

Online publiziert: 9. Juli 2020

(c) Der/die Autor(en) 2020

\section{Zusammenfassung}

Dieser Beitrag der Zeitschrift „Gruppe. Interaktion. Organisation. (GIO)“ beschäftigt sich mit der Prüfung und Validierung der Selbsteinschätzungsform des Deutschen Inventars Authentischer Führung.

Authentische Führung hat sich in den letzten Jahren als ein zentrales positives Führungskonzept herausgebildet. Allerdings liegt im deutschsprachigen Raum bisher nur ein Instrument zur Fremdeinschätzung vor: Das Deutsche Inventar authentischer Führung (DIAF-F). In diesem Beitrag wird ein Instrument zur Selbsteinschätzung authentischer Führung (DIAF-S) mit den Komponenten Selbstbewusstheit, Transparenz in Beziehungen, ausgewogene Informationsverarbeitung und verinnerlichte moralische Perspektive vorgestellt und erprobt. Zur ersten Validierung des neuen Verfahrens wurden Daten von $N=91$ Führungskräften und $N=259$ Mitarbeitenden erfasst. Für die Gesamtskala und drei der vier Komponenten ergaben sich interne Konsistenzen im befriedigenden bis guten Bereich. Das DIAF-S wies bedeutsame Zusammenhänge mit organisationalen Außenkriterien (durch Führungskraft eingeschätzte Arbeitsleistung der Mitarbeitenden, Voice-Verhalten und Moral Courage) auf. Zudem zeigte sich für einen Großteil der Komponenten die erwartete moderate Übereinstimmung zwischen selbst- und fremdeingeschätzter authentischer Führung. Mittels konfirmatorischer Faktorenanalysen ließ sich eine Verwendung der vierfaktoriellen Struktur analog zur Fremdbeurteilung rechtfertigen. Eine explorative Faktorenanalyse legte eher eine dreifaktorielle Struktur der selbsteingeschätzten authentischen Führung nahe. Vor diesem Hintergrund werden alternative Modelle authentischer Führung diskutiert. Insgesamt betrachtet stellt das DIAF-S ein weitgehend reliables und valides Instrument für die Selbsteinschätzung authentischer Führung und damit eine wichtige Ergänzung zum DIAF-F dar.

Schlüsselwörter Authentic Leadership Inventory · Authentische Führung · DIAF-F · Fragebogen · Fremdeinschätzung · Führungsstil $\cdot$ Selbsteinschätzung $\cdot$ Validität

Magdalena Reineboth

magdalena.reineboth@psychologie.tu-chemnitz.de

1 Professur für Persönlichkeitspsychologie und Diagnostik, Technische Universität Chemnitz, Wilhelm-Raabe-Straße

43, 09120 Chemnitz, Deutschland

2 Professur für Arbeits- und Organisationspsychologie,

Technische Universität Dresden, Zellescher Weg

17, 01069 Dresden, Deutschland 


\title{
Do I lead authentically? Validation of a self-rating version of the German Inventory of Authentic Leadership
}

\begin{abstract}
This paper of the magazine "Gruppe. Interaktion. Organisation. (GIO)" presents the psychometric properties and validation of the self-assessment form of the German Inventory of Authentic Leadership.

Authentic leadership has become one of the central positive leadership styles in recent years. Up to now, there has only been an other-rating version in German language to assess authentic leadership: The German Inventory of Authentic Leadership (DIAF-F). This article presents and tests the new instrument DIAF-S for self-assessment of authentic leadership with the components self-awareness, relational transparency, balanced processing and an internalized moral perspective. For first validation, the DIAF-S was tested on $N=91$ leaders and $N=265$ employees. The overall scale and three of four subscales showed satisfying to good internal consistencies. The DIAF-S correlated significantly with criteria of organizational performance (work performance of employees, voice behavior and moral courage rated by leader). In addition, for a majority of the components the expected moderate correlation between self- and other-ratings of authentic leadership was evident. The use of the four-factor structure could be verified by confirmatory factor analysis in analogy to the other-rating version of authentic leadership.

An explorative factor analysis revealed rather a three-factor structure of self-rated authentic leadership. In this context, we discuss alternative models of authentic leadership. Overall, the DIAF-S is a widely reliable and valid instrument for self-assessment of authentic leadership and thus an important supplement to the DIAF-F.
\end{abstract}

Keywords Authentic Leadership · Authentic Leadership Inventory · DIAF-F · Leadership style · Other-rating · Questionnaire $\cdot$ Self-report · validity

Das Große ist nicht, dies oder das zu sein, sondern man selbst zu sein. (Søren Kierkegaard)

Bereits Kierkegaard sah in der wahren Größe eines Menschen dessen Echtheit und das wahre Selbst zu offenbaren. Authentizität bedeutet, ein vertieftes Verständnis für das eigene Selbst zu haben und im Einklang mit seinen eigenen Gedanken, Gefühlen und Bedürfnissen zu agieren (Kernis 2003).

Authentisches Verhalten kann als wichtige Ressource für moralisches Verhalten verstanden werden (Hannah et al. 2011). Bei moralischen Belangen in Organisationen kommt Führungskräften eine tragende Rolle zu, da sie über ihre Vorbildwirkung das Verhalten von Mitarbeitenden beeinflussen (Treviño et al. 2014). Entsprechend kann authentisches Führungsverhalten durch Vorleben von Vertrauenswürdigkeit und moralischen Werten moralisches Verhalten von Mitarbeitenden fördern (Brown und Mitchell 2010).

\section{Authentische Führung}

Luthans und Avolio (2003) charakterisieren eine authentische Führungskraft als selbstsicher, transparent, auf Moral und Ethik orientiert und daran interessiert, Mitarbeitende selbst zu Führungskräften zu entwickeln. Aktuell wird authentische Führung definiert ,(...) as a pattern of leader behavior that draws upon and promotes both positive psychological capacities and a positive ethical climate, to foster greater self-awareness, an internalized moral perspective, balanced processing of information, and relational transparency on the part of leaders working with followers, fostering self-development" (Walumbwa et al. 2008, S. 94). Das Modell authentischer Führung nach Walumbwa et al. (2008) umfasst vier Verhaltenskomponenten: Selbstbewusstheit (SB) ist die Selbstreflexion der Führungskraft, die tiefgreifende Kenntnis eigener Stärken und Schwächen, das Antizipieren der eigenen Wirkung auf andere und das Einholen von Feedback; Transparenz in Beziehungen zu anderen $(\mathrm{TiB})$ umfasst, sein wahres Ich gegenüber anderen Personen zu zeigen, ohne dabei Gedanken oder Gefühle zu unterdrücken oder sich zu verstellen; Ausgewogene Informationsverarbeitung (AIV) erfasst die objektive Analyse relevanter Informationen beim Entscheidungsprozess, wobei Führungskräfte die Meinungen von Mitarbeitenden einholen und eigene Auffassungen zum Diskurs stellen; Verinnerlichte moralische Perspektive (VMP) beschreibt, wie sehr das Entscheiden und Handeln einer Führungskraft durch verinnerlichte moralische Standards und Werte gelenkt wird.

Angenommen wird, dass authentisches Führungsverhalten positive Effekte auf die Einstellungen und das Verhalten von Mitarbeitenden hat (Walumbwa et al. 2008). Zahlreiche Studien ermittelten positive Zusammenhänge zwischen authentischer Führung und behavioralen und affektiven Maßen wie beruflicher Leistung der Mitarbeitenden, Engagement, organizational citizenship behavior und Berufszufrie- 
denheit der Mitarbeitenden (Banks et al. 2016; Hoch et al. 2018).

\section{Das Deutsche Inventar Authentischer Führung (DIAF)}

\subsection{Das DIAF als Fremdeinschätzungsform (DIAF-F)}

Basierend auf dem Modell authentischer Führung (Walumbwa et al. 2008) wurde das Deutsche Inventar Authentischer Führung in der Fremdeinschätzungsform (DIAF-F, Franke-Bartholdt et al. 2018) als deutsche Übersetzung des Authentic Leadership Inventory (ALI, Neider und Schriesheim 2011) entwickelt (zur Verfahrensentwicklung des DIAF-F s. Franke-Bartholdt et al. 2018).

Die Güte und Validität des DIAF-F wurde in mehreren Studien geprüft (Franke-Bartholdt et al. 2018): Bei der Prüfung der internen Struktur mittels konfirmatorischer Faktorenanalyse ergab sich eine modellkonforme vierfaktorielle Struktur. Das Modell war einem Model mit vier Faktoren erster Ordnung und einem Faktor zweiter Ordnung (Gesamtwert authentischer Führung) sowie einem Modell mit einem Faktor überlegen. Die Überprüfung mit einer explorativen Faktorenanalyse ergab eine zweifaktorielle Lösung, bei welcher die Items der Komponenten SB, AIV und TiB auf Faktor 1 luden, die Items der Komponente VMP auf Faktor 2. Das DIAF-F korrelierte erwartungsgemäß hoch mit Facetten ethischer Führung (Brown et al. 2005). Hohe, negative Zusammenhänge zwischen dem Führungsstil Abusive Supervision (Tepper 2000) und dem DIAF-F belegten die Abgrenzung der beiden Führungsformen voneinander. Mit organisationalen Außenkriterien wie selbsteingeschätzter Leistung, Arbeitsengagement und Wohlbefinden der Mitarbeitenden ergaben sich positive, signifikante Assoziationen. Authentische Führung konnte über ethische Führung hinausgehend Varianz in den Kriterien Leistung und Leader-Member-Exchange aufklären.

\subsection{Das DIAF als Selbsteinschätzungsform (DIAF-S): Ziele und Hypothesen}

Insbesondere bei der Bewertung des eigenen Führungsstils tendieren Führungskräfte zu einer überhöhten Selbsteinschätzung (Yammarino und Atwater 1997), so dass das Vorliegen eines Testverfahrens als Selbst-und Fremdeinschätzungsform besonders im Bereich des Führungsverhaltens einen Mehrwert liefert. Daher soll mit der vorliegenden Arbeit das DIAF-S als Ergänzung zum DIAF-F umfassend geprüft und validiert werden.

Das DIAF-S wurde aus dem DIAF-F (Franke-Bartholdt et al. 2018) abgeleitet, indem die Items in die Ich-Perspektive transformiert wurden. Das DIAF-S besteht aus 16 Items und bildet die vier Komponenten authentischer Führung Selbstbewusstheit (z. B. „Ich bin mir selbst klar bewusst, welche Wirkung ich auf andere habe."), Transparenz in Beziehungen zu anderen (z.B. „Ich sage offen, wie ich mich wirklich fühle, und verstelle mich nicht. "), verinnerlichte moralische Perspektive (z. B. ,Ich handele in Übereinstimmung mit meinen Überzeugungen und Werten.") und ausgewogene Informationsverarbeitung (z. B. „Ich frage aktiv auch nach Standpunkten die meine Überzeugungen in Frage stellen.") mit je vier Items ab. Die Items sind auf einer fünf-stufigen Likertskala (von 1=stimme gar nicht $z u$ bis $5=$ stimme vollkommen $\mathrm{zu}$ ) zu beantworten.

Um Auskunft über die Eignung der einzelnen Items für die Komponenten und die Gesamtskala zu erhalten, wurden die psychometrischen Eigenschaften des DIAF-S geprüft. Zur Ermittlung der Konstruktvalidität wurde die faktorielle Binnenstruktur mittels konfirmatorischer und explorativer Faktorenanalyse bestimmt. Für die Vergleichbarkeit des DIAF-S mit dem DIAF-F wurde zusätzlich die faktorielle Validität des DIAF-F bestimmt.

Zur Prüfung der Kriteriumsvalidität wurden Zusammenhänge mit Leistung und pro-organisationalem Verhalten, operationalisiert als Voice (Liang et al. 2012) und Moral Courage (Hannah et al. 2011), herangezogen. Hierbei sollen folgende Hypothesen überprüft werden.

Authentische Führungskräfte befördern die Selbstbestimmung und Selbstregulation ihrer Mitarbeitenden, sodass dies die Motivation und Zufriedenheit der Mitarbeitenden erhöht, was wiederum positive Effekte auf die Arbeitsleistung hat (Leroy et al. 2015). Mehrfach wurde der positive Zusammenhang zwischen authentischer Führung und Leistung repliziert (Hoch et al. 2018; Banks et al. 2016). Empirisch zeigte sich über verschiedene Studien hinweg ein kleiner Effekt $(\rho=0,14)$ zwischen authentischer Führung und Leistung der Mitarbeitenden. Entsprechend ergibt sich:

Hypothese 1 Es besteht ein niedriger positiver Zusammenhang zwischen selbsteingeschätzter authentischer Führung und der Leistung der Mitarbeitenden.

Authentische Führungskräfte fungieren als Rollenmodelle und spiegeln Integrität, Ehrlichkeit und hohe moralische Standards in der Beziehung zu Mitarbeitenden wider (Brown und Mitchell 2010). Durch ihre Vorbildwirkung befördern sie soziale Lernprozesse der Mitarbeitenden und formen den normativen Kontext für moralisches Handeln. Entsprechend können authentische Führungskräfte moralische Einstellungen und Verhaltensweisen ihrer Mitarbeitenden beeinflussen, die die Funktionsfähigkeit der Organisation unterstützen (Treviño et al. 2014). So befördern authentische Führungskräfte, dass die Mitarbeitenden selbst eher bereit sind, Veränderungen anzustoßen und ein sogenann- 
tes Voice-Verhalten (Wong und Cummings 2009) zu zeigen, und an moralischen Standards in Form von Moral Courage festzuhalten (Hannah et al. 2011). Liang et al. (2012) unterscheiden zwischen prohibitive Voice, dem aktiven Ansprechen von Problemen in der Arbeitsumgebung und promotive Voice, dem Äußern von Ideen zur Verbesserung von Arbeitsprozessen. Empirisch konnte gezeigt werden, dass authentische Führung einen indirekten Effekt, vermittelt über Vertrauen in die Führungskraft, auf das Voice-Verhalten der Mitarbeitenden hat (Wong und Cummings 2009). Metaanalytisch wurde ein Zusammenhang von $\rho=0,31$ zwischen authentischer Führung und Voice-Verhalten gefunden (Banks et al. 2016). Zwischen authentischer Führung und Moral Courage konnte ein Zusammenhang im mittleren Bereich $(r=0,29)$ gefunden werden (Hannah et al. 2011). Es ergeben sich folgende Hypothesen:

Hypothese 2a und 2b Es besteht ein moderater positiver Zusammenhang zwischen selbsteingeschätzter authentischer Führung und dem promotive-Voice- bzw. prohibitiveVoice-Verhalten der Mitarbeitenden.

Hypothese 2c Es besteht ein moderater positiver Zusammenhang zwischen selbsteingeschätzter authentischer Führung und Moral Courage der Mitarbeitenden.

Als weiteres Kriterium wird die Fremdeinschätzung der Mitarbeitenden herangezogen. Insbesondere besteht bei der Einschätzung des eigenen Führungsstils ein Risiko zur Selbstüberschätzung (Yammarino und Atwater 1997). In einer Metanalyse von Lee und Carpenter (2018) wurde die Übereinstimmung zwischen Selbst- und Fremdeinschätzung für verschiedene Führungsstile geprüft: Für beziehungsorientierte Führungsstile, zu denen auch authentische Führung gezählt werden kann (Lee und Carpenter 2018), fand sich eine Korrelation von $\rho=0,27$, was $\mathrm{zu}$ folgender Annahme führt:

Hypothese 3 Es besteht ein moderater positiver Zusammenhang zwischen selbst- und fremdeingeschätzter authentischer Führung.

\section{Methoden}

\subsection{Stichprobe}

An der Studie nahmen $N=91$ Führungskräfte $(68,5 \%$ weiblich) aus vier Organisationen des Gesundheitssektors teil (Alter: $M=45,43, S D=9,61$, Range 26-61). Von den Befragten waren $31,1 \%$ weniger als fünf Jahre, 38,9\% zwischen 6 und 20 Jahren und 30,0\% mehr als 20 Jahre im aktuellen Arbeitsfeld tätig. Die Rekrutierung der
Teilnehmenden erfolgte im Rahmen des DFG-Projekts FIDES (Führung in Organisationen als Determinante des Schweigens von Mitarbeitern zu moralisch problematischen Sachverhalten) in welchem der Zusammenhang zwischen (un)moralischen Führungsformen und dem Verhalten von Mitarbeitenden in moralisch relevanten Situationen untersucht wird. Für das Projekt (GZ STR 1005/5-1; WE 1504/25-1) wurde ein positives Ethikvotum erteilt. Die Fragebögen wurden mittels Onlineumfrage oder paperpencil Variante ausgefüllt. Zur Überprüfung der faktoriellen Validität des DIAF-S wurde nach Ausschluss von Führungskräften mit fehlenden Werten eine Stichprobe von $n=80$ Führungskräften $(66,7 \%$ weiblich, Alter: $M=44,86$, $\mathrm{S} D=9,65)$ genutzt, für das DIAF-F wurde auf die korrespondierende Gesamtstichprobe der Mitarbeitenden zurückgegriffen, welche nach Ausschluss von Mitarbeitenden mit fehlenden Werten ein $n=620$ (76,1\% weiblich, Alter: $M=42,21, \mathrm{~S} D=12,32$ ) ergab.

Zur Bestimmung des Zusammenhangs zwischen Fremdund Selbsteinschätzung authentischer Führung wurde eine Stichprobe von $n=42$ Führungskräften $(66,7 \%$ weiblich, Alter: $M=44,00, \mathrm{~S} D=8,78)$ und $n=259$ Mitarbeitenden (76,8\% weiblich, Alter: $M=40,02, \mathrm{~S} D=10,98)$ genutzt.

\subsection{Instrumente}

In Tab. 4 sind die internen Konsistenzen der verwendeten Verfahren dargestellt. Die Selbsteinschätzung authentischen Führungsverhalten wurde mittels DIAF-S anhand von je vier Items pro Komponente auf einer fünf-stufigen Likertskala (von $1=$ stimme gar nicht $z u$ bis $5=$ stimme vollkommen zu) erfasst.

Für die Fremdeinschätzung authentischer Führung wurde das DIAF-F genutzt (Franke-Bartholdt et al. 2018), welches analog zu dem DIAF-S aus 16 Items besteht (z.B. „Mein direkter Vorgesetzter/Meine direkte Vorgesetzte hört sich alternative Standpunkte sorgfältig an, bevor er/sie Schlüsse zieht.“). Die Items wurden auf einer fünf-stufigen Skala beantwortet (von $1=$ stimme gar nicht $z u$ bis $5=$ stimme vollkommen $z u)$.

Voice wurde mit der deutschen Übersetzung (Rose 2015) des Fragebogens von Liang et al. (2012) erfasst, in dem promotive und prohibitive Voice jeweils über fünf Items erfragt werden (promotive Voice, z. B. „Die Mitarbeiter(innen) meines Arbeitsbereichs schlagen aktiv neue Projekte vor, die nutzbringend für den Arbeitsbereich sind“; prohibitive Voice, z. B. „Die Mitarbeiter(innen) meines Arbeitsbereichs trauen sich, auf Probleme im Arbeitsbereich hinzuweisen, auch wenn das die Beziehung $\mathrm{zu}$ anderen Kolleg(inn)en beeinträchtigen könnte.“). Die Einschätzung des Voice-Verhaltens erfolgte auf einer fünf-stufigen Skala (von $1=$ stimme gar nicht $z u$ bis $5=$ stimme vollkommen $z u$ ). 
Moral Courage wurde über sechs Items des Moral Potency Questionnaire von Hannah und Avolio (2010) in der deutschen Übersetzung von Rose (2015) erfasst. Die Führungskräfte schätzten ihre Mitarbeitenden hinsichtlich des Festhaltens an moralischen Prinzipien auf einer fünfstufigen Likerstskala ein (von 1= stimme gar nicht $z u$ bis $5=$ stimme vollkommen $z u$; z.B. „Die Mitarbeiter(innen) meines Arbeitsbereichs übernehmen Verantwortung und greifen ein, wenn sie eine moralisch problematische Handlung beobachten.").

Die Arbeitsleistung wurde über zwei Items erfasst (Robinson 1996): Die Führungskraft beurteilte die Leistung der eigenen Mitarbeitenden im Vergleich zu anderen Teams und gab weiterhin an, wie die Mitarbeitenden vermutlich ihre eigene Leistung im Vergleich zu der anderer Teams einstufen würde. Die Einschätzung erfolgte auf einer sieben-stufigen Likertskala $(1=$ deutlich schlechter bis $7=$ deutlich besser $)$. Da beide Items hoch miteinander korrelierten $(r=0,58$, $p<0,001)$ wurde ein Gesamtwert für Arbeitsleistung gebildet.

\subsection{Analysen}

Die Auswertung der Daten erfolgte mit dem Statistikprogramm IBM SPSS Statistics (Version 25.0., 2017). Eine Ausreißeranalyse ergab keine multivariaten Ausreißer. Ver- einzelt traten fehlende Werte auf, die durch einen paarweisen Ausschluss berücksichtigt wurden.

Zur Prüfung der faktoriellen Validität des DIAF-S wurden mittels konfirmatorischer Faktorenanalysen (CFA) verschiedene, theoretisch abgeleitete Modelle getestet. Für die Vergleichbarkeit des DIAF-S mit dem DIAF-F bei der Bestimmung des Zusammenhangs zwischen selbst- und fremdeingeschätzter authentischer Führung, wurde zusätzliche die faktorielle Validität des DIAF-F bestimmt. Die Berechnung erfolgte mit dem Programm IBM SPSS AMOS (Version 26,0; Arbuckle 2014). Zur Prüfung des globalen Modell-Fits wurde unter Verwendung der Maximum-Likelihood Schätzmethode der $\chi^{2}$-Test herangezogen. Zur Beurteilung des Gesamt-Fits eines Modells empfehlen Hu und Bentler (1999) bei geringem Stichprobenumfang $(N \leq 250)$ zusätzlich die Kombination aus verschiedenen Fit-Indizes: Comparative Fit Index (CFI), Root Mean Square Error of Approximation (RMSEA) und Standardized Root Mean Square Residual (SRMR). Zur Interpretation der Indizes wurden die von Hu und Bentler (1999) vorgeschlagenen Cut-Off-Werte genutzt: $\mathrm{CFI} \geq 0,95$, RMSEA $<0,06$ (bei $N \geq 250$ ) bzw. $<0,08$ (bei $N \leq 250$ ) und $\mathrm{SRMR}<0,09$. Zudem wurden die Modelle mittels $\chi^{2}$-Differenz-Test miteinander verglichen.

Zusätzlich wurde für eine differenzierte Erfassung der faktoriellen Binnenstruktur des DIAF-S eine explorative Faktorenanalyse (EFA) durchgeführt. Aufgrund der Annah-
Tab. 1 Itemkennwerte des DIAF-S

\begin{tabular}{|c|c|c|c|c|c|c|c|c|c|}
\hline Item & $n$ & Min & Max & $M$ & $S D$ & $r_{\mathrm{it}-\mathrm{G}}$ & $r_{\mathrm{it}-\mathrm{K}}$ & Skew & Kurt \\
\hline \multicolumn{10}{|c|}{ Selbstbewusstheit (SB) } \\
\hline 1 & 90 & 2,00 & 5,00 & 4,22 & 0,87 & 0,30 & 0,22 & $-0,87$ & $-0,10$ \\
\hline 5 & 90 & 1,00 & 5,00 & 3,26 & 0,74 & 0,62 & 0,51 & 0,22 & 0,82 \\
\hline 8 & 91 & 1,00 & 5,00 & 4,44 & 0,69 & 0,28 & 0,28 & $-1,67$ & 5,63 \\
\hline 12 & 90 & 2,00 & 5,00 & 3,82 & 0,77 & 0,50 & 0,38 & $-0,13$ & $-0,47$ \\
\hline \multicolumn{10}{|c|}{ Transparenz in Beziehungen (TiB) } \\
\hline 2 & 91 & 1,00 & 5,00 & 4,14 & 0,75 & 0,47 & 0,59 & $-0,88$ & 1,96 \\
\hline 9 & 90 & 3,00 & 5,00 & 4,42 & 0,62 & 0,60 & 0,47 & $-0,58$ & $-0,57$ \\
\hline 13 & 90 & 3,00 & 5,00 & 4,21 & 0,66 & 0,49 & 0,67 & $-0,26$ & $-0,73$ \\
\hline 16 & 91 & 1,00 & 5,00 & 3,82 & 0,97 & 0,47 & 0,60 & $-0,82$ & 0,73 \\
\hline \multicolumn{10}{|c|}{ Verinnerlichte moralische Perspektive (VMP) } \\
\hline 3 & 90 & 3,00 & 5,00 & 4,44 & 0,69 & 0,38 & 0,48 & $-0,85$ & $-0,46$ \\
\hline 6 & 91 & 2,00 & 5,00 & 4,42 & 0,68 & 0,30 & 0,40 & $-0,97$ & 0,63 \\
\hline 10 & 90 & 1,00 & 5,00 & 3,81 & 0,89 & 0,38 & 0,39 & $-0,31$ & $-0,14$ \\
\hline 14 & 90 & 3,00 & 5,00 & 4,47 & 0,60 & 0,38 & 0,51 & $-0,65$ & $-0,50$ \\
\hline \multicolumn{10}{|c|}{ Ausgewogene Informationsverarbeitung (AIV) } \\
\hline 4 & 87 & 1,00 & 5,00 & 4,00 & 0,81 & 0,40 & 0,41 & $-1,09$ & 2,77 \\
\hline 7 & 91 & 3,00 & 5,00 & 4,34 & 0,67 & 0,42 & 0,63 & $-0,53$ & $-0,72$ \\
\hline 11 & 91 & 2,00 & 5,00 & 4,12 & 0,76 & 0,40 & 0,45 & $-0,52$ & $-0,15$ \\
\hline 15 & 91 & 3,00 & 5,00 & 4,53 & 0,66 & 0,43 & 0,43 & $-1,07$ & 0,01 \\
\hline
\end{tabular}

Die Items werden auf einer fünf-stufigen Skala beantwortet $(1=$ stimme gar nicht $\mathrm{zu}, 2=$ stimme eher nicht $\mathrm{zu}, 3=$ teils/teils, $4=$ stimme eher zu, 5=stimme vollkommen $\mathrm{zu}$ ). $r_{i t-G}=$ Trennschärfe bezogen auf die Gesamtskala; $r_{i t-K}=$ Trennschärfe bezogen auf die Komponente, aus der ein Item stammt; Skew $=$ Schiefe, Kurt $=$ Exzess 
Tab. 2 Ergebnisse der konfirmatorischen Faktorenanalysen für DIAF-S und DIAF-F

\begin{tabular}{|c|c|c|c|c|c|c|c|c|c|c|}
\hline & \multirow[b]{2}{*}{ Modell } & \multicolumn{3}{|l|}{$\chi^{2}$ Test $^{\mathrm{a}}$} & \multirow[b]{2}{*}{ CFI } & \multirow[b]{2}{*}{ RMSEA } & \multirow[b]{2}{*}{ SRMR } & \multicolumn{3}{|c|}{$\chi^{2}$ Differenz-Test } \\
\hline & & $\overline{\chi^{2}}$ & $d f$ & $p$ & & & & $\overline{\Delta \chi^{2}}$ & $\Delta d f$ & $p$ \\
\hline \multirow[t]{4}{*}{$\overline{\text { DIAF-S }}$} & 1 & 138,65 & 98 & 0,004 & 0,86 & 0,07 & 0,08 & - & - & - \\
\hline & 2 & 140,28 & 100 & 0,005 & 0,86 & 0,07 & 0,08 & 1,63 & 2 & 0,443 \\
\hline & 3 & 171,85 & 103 & 0,001 & 0,76 & 0,09 & 0,09 & 33,20 & 5 & $<0,001$ \\
\hline & 4 & 199,48 & 104 & 0,001 & 0,67 & 0,11 & 0,09 & 60,83 & 6 & $<0,001$ \\
\hline \multirow[t]{4}{*}{ DIAF-F } & 1 & 368,91 & 98 & 0,001 & 0,96 & 0,07 & 0,04 & - & - & - \\
\hline & 2 & 397,65 & 100 & 0,001 & 0,96 & 0,07 & 0,04 & 27,74 & 2 & $<0,001$ \\
\hline & 3 & 466,27 & 103 & 0,001 & 0,95 & 0,08 & 0,04 & 97,36 & 5 & $<0,001$ \\
\hline & 4 & 772,38 & 104 & 0,001 & 0,90 & 0,10 & 0,06 & 403,47 & 6 & $<0,001$ \\
\hline
\end{tabular}

Modell 1 = vier interkorrelierte Faktoren; Modell 2= vier Faktoren erster Ordnung, ein Faktor zweiter Ordnung; Modell 3= zwei Faktoren; Modell $4=$ ein Faktor. ${ }^{a} p$-Wert nach Bollen-Stine-Bootstrap-Korrektur. Aufgrund einzelner fehlender Werte lag der Stichprobenumfang für DIAF-S bei $n=80$ Führungskräften. Der Stichprobenumfang für DIAF-F lag bei $n=620$ Mitarbeitenden

CFI Comparative Fit Index, RMSEA Root Mean Square Error of Approximation, SRMR Standardized Root Mean Residual

me korrelierender Faktoren wurde eine Hauptachsenanalyse mit obliquer Rotation (Promax, Kappa $=4$ ) angewandt. Das Extraktionskriterium für die Anzahl der Faktoren war die Parallelanalyse nach Horn (1965; umgesetzt mit O'Connor 2000).

Zur Prüfung der Kriteriumsvalidität wurden korrelative Zusammenhänge zwischen DIAF-S und organisationalen Außenkriterien betrachtet. Aufgrund moderater Zusammenhänge zwischen dem DIAF-S mit Alter und Tätigkeitsdauer der Führungskräfte wurden Zusammenhänge mit Außenkriterien mittels Partialkorrelationen bestimmt. Das $\alpha$-Niveau wurde Bonferroni-korrigiert, was zu einem $\alpha^{\prime}=0,05 / 9 \approx 0,006$ führte. Um den Vergleich zwischen DIAF-S und DIAF-F zu ermöglichen, wurde zur Berechnung der Kriteriumsvalidität auf die vierfaktorielle Struktur authentischer Führung zurückgegriffen.

Für die Bestimmung des Zusammenhangs zwischen fremd- und selbsteingeschätzter authentischer Führung wurden die Angaben der Führungskräfte einbezogen, für die eine direkte Zuordnung zu ihren Mitarbeitenden aufgrund des Teamcodes möglich war. Pro Führungskraft wurde der Mittelwert über alle Fremdeinschätzungen gebildet und mit der Selbsteinschätzung korreliert. Hierfür standen die Datensätze von $n=42$ Führungskräften und $n=259$ Mitarbeitenden zur Verfügung. Für jede Führungskraft lagen zwischen 2 und 15 Fremdeinschätzungen vor $(M d n=6)$. Um die Güte der Interrater-Übereinstimmung beurteilen zu können, wurde für jedes Team der $r_{W G}$-Wert (withingroup correlation) berechnet. Um verschiedene Antworttendenzen bei der Beurteilung durch die Mitarbeitenden zu berücksichtigen, wurden Werte für die Annahme eines gleichverteilten Antwortmusters (stetige Gleichverteilung) und einer Antworttendenz, die dem Lenciency Bias unterliegt (Verhalten wird tendenziell besser eingeschätzt, als es eigentlich ist; leicht schiefe Verteilungsannahme) berechnet (LeBreton und Senter 2008). Das $\alpha$-Niveau wurde für die Zusammenhänge zwischen selbst- und fremdeingeschätzter authentischer Führung Bonferroni-korrigiert, was zu einem $\alpha^{\prime}=0,05 / 10 \approx 0,005$ führte.

\section{Ergebnisse}

\subsection{Deskriptive Statistik der Items, Komponenten und Gesamtskala}

Tab. 1 stellt relevante Kennwerte (deskriptive Statistik, Trennschärfen) der 16 Items dar; Tab. 4 die deskriptiven Statistiken der Komponenten und der Gesamtskala ebenso wie die Korrelationen zwischen demographischen Variablen und DIAF-S. Aufgrund der beobachteten $\mathrm{Zu}$ sammenhänge wurden das Alter und die Tätigkeitsdauer als Kontrollvariablen in die folgenden Berechnungen der Zusammenhänge zwischen dem DIAF-S und den Kriterien einbezogen.

Die Itemschwierigkeiten lagen im niedrigen Bereich $(3,26 \leq M \leq 4,53)$. Die Schwierigkeiten der Komponenten und der Gesamtskala waren mit Werten zwischen 3,94 (SB) und 4,28 (VMP) ebenfalls niedrig. Alle Items wiesen eine Schiefe $<|2|$ und Kurtosis $<17 \mid$ auf, was eine Normalverteilung nahelegt (West et al. 1995). Für alle Items ergab sich eine linksschiefe, rechtssteile Verteilung.

Die Trennschärfen lagen überwiegend im akzeptablen Bereich: Bezogen auf die Gesamtskala im Bereich von $0,28<r_{\text {it-G }}<0,62$, für die jeweiligen Komponenten im Bereich von $0,22<r_{\mathrm{it}-\mathrm{K}}<0,67$. Auffällig waren die geringen Trennschärfen von zwei Items der Komponente SB mit Werten $r_{\mathrm{it}-\mathrm{K}}<0,30$ sowie die geringen Trennschärfen der Items der Komponente VMP $\left(r_{\mathrm{it}-\mathrm{G}}<0,40\right)$ (Tab. 1).

\subsection{Reliabilität}

Die interne Konsistenz der Gesamtskala des DIAF-S ist mit einem Cronbachs Alpha von 0,82 als gut einzustufen. 
Tab. 3 Faktorladungen der Items auf Basis der explorativen Faktorenanalyse

\begin{tabular}{|c|c|c|c|c|}
\hline Item & Itemwortlaut & $\begin{array}{l}\text { Faktor } 1 \\
\lambda\end{array}$ & $\begin{array}{l}\text { Faktor } 2 \\
\lambda\end{array}$ & $\begin{array}{l}\text { Faktor } 3 \\
\lambda\end{array}$ \\
\hline \multicolumn{5}{|c|}{$\overline{\text { Selbstbewusstheit (SB) }}$} \\
\hline 1 & Ich erbitte Rückmeldung, um meinen Umgang mit anderen zu verbessern & 0,16 & $\mathbf{0 , 3 1}$ & $-0,13$ \\
\hline 5 & $\begin{array}{l}\text { Ich kann genau beschreiben, wie meine Fähigkeiten durch andere beurteilt wer- } \\
\text { den }\end{array}$ & 0,63 & 0,10 & 0,04 \\
\hline 8 & Ich verstehe, wo meine Stärken und Schwächen liegen & $\mathbf{0 , 3 7}$ & 0,11 & $-0,12$ \\
\hline 12 & Ich bin mir selbst klar bewusst, welche Wirkung ich auf andere habe & $\mathbf{0 , 3 6}$ & 0,15 & 0,17 \\
\hline \multicolumn{5}{|c|}{ Transparenz in Beziehungen zu anderen (TiB) } \\
\hline 2 & Ich sage offen das, was ich wirklich denke, sodass andere wissen, woran man ist & $\mathbf{0 , 7 0}$ & $-0,09$ & 0,03 \\
\hline 9 & $\begin{array}{l}\text { Ich schaffe Transparenz und Klarheit, indem ich andere offen über Anliegen } \\
\text { informiere, die mir wichtig sind }\end{array}$ & $\mathbf{0 , 5 5}$ & 0,30 & $-0,09$ \\
\hline 13 & $\begin{array}{l}\text { Ich bringe anderen gegenüber klar zum Ausdruck, welche Gedanken und Ideen } \\
\text { ich habe }\end{array}$ & $\mathbf{0 , 5 9}$ & 0,05 & 0,07 \\
\hline 16 & Ich sage offen, wie ich mich wirklich fühle, und verstelle mich nicht & 0,75 & $-0,15$ & 0,06 \\
\hline \multicolumn{5}{|c|}{ Verinnerlichte moralische Perspektive (VMP) } \\
\hline 3 & Ich handele in Übereinstimmung mit meinen Überzeugungen und Werten & 0,01 & $-0,18$ & 0,76 \\
\hline 6 & $\begin{array}{l}\text { Ich stütze mich auf meine Überzeugungen und Werte, um wichtige Entschei- } \\
\text { dungen zu treffen }\end{array}$ & $-0,02$ & 0,13 & $\mathbf{0 , 4 2}$ \\
\hline 10 & $\begin{array}{l}\text { Ich widersetze mich, wenn ich zu Dingen gedrängt werde, die im Widerspruch } \\
\text { zu meinen Überzeugungen und Werten stehen }\end{array}$ & 0,19 & 0,03 & $\mathbf{0 , 3 2}$ \\
\hline 14 & $\begin{array}{l}\text { Ich richte meine Handlungen nach meinen moralischen Überzeugungen und } \\
\text { Werten aus }\end{array}$ & 0,00 & 0,06 & 0,66 \\
\hline \multicolumn{5}{|c|}{ Ausgewogene Informationsverarbeitung (AIV) } \\
\hline 4 & $\begin{array}{l}\text { Ich frage aktiv auch nach Standpunkten, die meine Überzeugungen in Frage } \\
\text { stellen }\end{array}$ & 0,06 & 0,34 & 0,20 \\
\hline 7 & Ich höre mir alternative Standpunkte sorgfältig an, bevor ich Schlüsse ziehe & $-0,25$ & $\mathbf{0 , 8 9}$ & 0,14 \\
\hline 11 & $\begin{array}{l}\text { Ich analysiere erst objektiv alle relevanten Informationen, bevor ich eine Ent- } \\
\text { scheidung treffe }\end{array}$ & 0,11 & 0,61 & $-0,07$ \\
\hline 15 & Ich ermutige andere, offen auch gegensätzliche Sichtweisen zu äußern & 0,16 & $\mathbf{0 , 5 1}$ & $-0,05$ \\
\hline
\end{tabular}

Fett hervorgehobene Werte kennzeichnen Hauptladungen

Die internen Konsistenzen der Komponenten lagen im Bereich von $0,55(\mathrm{SB}) \leq \alpha \leq 0,77$ (TiB). Für die Komponenten TiB, VMP und AIV lassen sich unter Berücksichtigung der geringen Itemanzahl die internen Konsistenzen als befriedigend bis gut einstufen. Die Komponente SB fiel durch eine niedrige interne Konsistenz auf, die als unzureichend eingestuft wird.

\subsection{Faktorielle Validität}

Für die Überprüfung der faktoriellen Binnenstruktur des DIAF-S wurden in CFA vier Modelle gegeneinander getestet: (1) Ein Modell mit vier interkorrelierten Faktoren, die die Komponenten authentischer Führung abbilden (Walumbwa et al. 2008), (2) ein Modell mit vier Faktoren erster Ordnung und einem Faktor zweiter Ordnung (Gesamtwert authentische Führung), (3) ein Modell mit zwei interkorrelierten Faktoren entsprechend der Befunde der explorativen Faktorenanalyse des DIAF-F (Franke-Bartholdt et al. 2018) und (4) ein Modell mit einem Globalfaktor authentischer Führung, auf dem alle 16 Items luden. Um die Vergleich- barkeit des DIAF-S mit dem DIAF-F zu gewährleisten, wurden alle vier Modelle ebenfalls für das DIAF-F getestet. Die Ergebnisse sind Tab. 2 zu entnehmen.

Für das DIAF-S erwiesen sich das Modell 1 mit vier interkorrelierten Faktoren $(\bar{r}=0,50)$ und das Modell 2 mit vier Faktoren erster Ordnung und einem Faktor zweiter Ordnung am ehesten geeignet, um die Daten der Selbsteinschätzung authentischer Führung abzubilden. Modell 1 und Modell 2 unterschieden sich nicht signifikant voneinander, auch die verschiedenen Fit-indizes unterschieden sich nicht in ihrer Höhe. Die Fit-Indizes RMSEA und SRMR lagen unter den Cutoff-Werten von Hu und Bentler (1999), der CFI-Wert erreichte allerdings für keines der beiden Modelle den gewünschten Cutoff-Wert. Beide Modelle erklärten die Datenstruktur signifikant besser auf als Modell 3 und 4. Da sparsamere Modelle komplexeren Modellen vorzuziehen sind (Steiger 1990), wurde das weniger komplexe Modell 1 mit vier interkorrelierten Faktoren fokussiert. Bei Betrachtung der Modifikationsindizes fiel auf, dass zwischen elf Items spezifizierte Korrelationen zwischen den Fehlervarianzen zu einem besseren Modell-Fit führen würden. 
Tab. 4 Deskriptive Statistiken, Reliabilitäten und Interkorrelationen der eingesetzten Skalen

\begin{tabular}{|c|c|c|c|c|c|c|c|c|c|c|c|c|c|c|c|c|c|}
\hline & $n$ & Min & Max & $M$ & $S D$ & 1 & 2 & 3 & 4 & 5 & 6 & 7 & 8 & 9 & 10 & 11 & 12 \\
\hline 1. Geschlecht & 89 & 1,00 & 2,00 & 1,69 & 0,47 & - & & & & & & & & & & & \\
\hline 2. Alter & 89 & 26,00 & 61,00 & 45,43 & 9,61 & 0,10 & - & & & & & & & & & & \\
\hline 3. TD & 90 & 1,00 & 3,00 & 1,99 & 0,79 & 0,11 & $0,58 * *$ & - & & & & & & & & & \\
\hline 4. SB & 91 & 2,50 & 5,00 & 3,94 & 0,51 & 0,11 & $0,31 * *$ & 0,17 & $(0,55)$ & & & & & & & & \\
\hline 5. TiB & 91 & 2,75 & 5,00 & 4,15 & 0,59 & 0,23 * & $0,37 * *$ & $0,35^{*}$ & $* 0,48 * *$ & $(0,77)$ & & & & & & & \\
\hline 6. VMP & 91 & 3,00 & 5,00 & 4,28 & 0,50 & $-0,08$ & $0,48 * *$ & $0,21 *$ & 0,21 & $0,22 *$ & $(0,65)$ & & & & & & \\
\hline 7. AIV & 91 & 2,25 & 5,00 & 4,26 & 0,53 & 0,19 & $0,26^{*}$ & 0,05 & $0,38 * *$ & $0,34 * *$ & 0,13 & $(0,69)$ & & & & & \\
\hline $\begin{array}{l}\text { 8. DIAF-S } \\
\text { Gesamt }\end{array}$ & 91 & 3,06 & 5,00 & 4,16 & 0,39 & 0,15 & $0,46 * *$ & $0,30 *$ & $* 0,75 * *$ & $0,77 * *$ & $0,54 * *$ & $0,68 * *$ & $(0,82)$ & & & & \\
\hline $\begin{array}{l}\text { 9. Promotive } \\
\text { Voice }\end{array}$ & 91 & 1,60 & 5,00 & 3,84 & 0,76 & 0,17 & 0,20 & 0,06 & $0,44 * *$ & $0,32 * *$ & 0,15 & $0,29 *$ & $0,44 * *$ & $(0,90)$ & & & \\
\hline $\begin{array}{l}\text { 10. Prohibitive } \\
\text { Voice }\end{array}$ & 91 & 2,20 & 5,00 & 3,61 & 0,71 & 0,05 & 0,10 & 0,02 & $0,50 * *$ & $0,36^{* *}$ & 0,15 & $0,28 *$ & $0,47 * *$ & $0,79 * *$ & $(0,85)$ & & \\
\hline $\begin{array}{l}\text { 11. Moral } \\
\text { Courage }\end{array}$ & 87 & 1,83 & 5,00 & 3,51 & 0,71 & $-0,07$ & $0,21 *$ & $-0,02$ & $0,29 *$ & $0,31 * *$ & 0,09 & $0,29 * *$ & $0,36 * *$ & $0,58 * *$ & $0,66^{* *}$ & $(0,87)$ & \\
\hline 12. Leistung & 86 & 3,00 & 7,00 & 4,98 & 0,91 & 0,07 & 0,05 & 0,06 & 0,11 & $0,25^{*}$ & $0,29 *$ & 0,18 & $0,30 *$ & 0,16 & $0,30 *$ & 0,15 & $(0,74)$ \\
\hline
\end{tabular}

Partialkorrelationen (Kontrollvariablen: Alter, Tätigkeitsdauer). In den Klammern der Diagonalen sind die internen Konsistenzen (Cronbachs Alpha) der (Sub-)Skalen angegeben. Es ergeben sich durch einzelne fehlende Werte unterschiedliche Stichprobengrößen. Kodierung Geschlecht: $1=$ männlich, 2 = weiblich. Kodierung Tätigkeitsdauer: $1=$ bis 5 Jahre, $2=6$ bis 20 Jahre, $3=$ länger als 20 Jahre

$T D$ Tätigkeitsdauer, $S B$ Selbstbewusstheit, TiB Transparenz in Beziehungen zu anderen, VMP verinnerlichte moralische Perspektive, $A I V$ ausgewogene Informationsverarbeitung, DIAF-S Deutsches Inventar Authentischer Führung als Selbsteinschätzungsform

*Die Korrelation ist auf dem Niveau von 0.05 signifikant (2-seitig); **Die Korrelation ist auf dem Niveau von 0,006 signifikant (2-seitig,

Bonferroni-korrigiertes Niveau)

Eine EFA mittels Parallelanalyse ergab für das DIAF-S drei Faktoren, welche 37,96\% der Varianz aufklärten und moderat miteinander korrelierten $(0,32<r<0,44)$. Tab. 3 sind die Faktorladungen zu entnehmen. Alle vier Items der Komponente TiB sowie drei Items der Komponente SB luden auf Faktor 1. Die Items der Komponente AIV luden gemeinsam mit Item 1 der Komponente SB auf Faktor 2. Alle Items der Komponente VMP wiesen die höchsten Ladungen auf Faktor 3 auf und zeigten somit eine Einfachstruktur auf.

Für das DIAF-F zeigte das Modell 1 mit vier interkorrelierten Faktoren $(\bar{r}=0,85)$ den besten Modell-Fit. Die FitIndizes RMSEA und SRMR lagen unter, der Index CFI über den von Hu und Bentler (1999) empfohlenen Cut-OffWerten. Modell 1 erklärte die Datenstruktur des DIAF-F signifikant besser auf als die drei Alternativmodelle, wobei Modell 2 mit vier Faktoren erster Ordnung und einem Faktor zweiter Ordnung und Modell 3 mit zwei interkorrelierten Faktoren ebenfalls zufriedenstellende Fit-Indizes aufwiesen (Tab. 2).

\subsection{Zusammenhänge zu Kriterien}

Die Korrelationen zwischen den Außenkriterien Arbeitsleistung, Voice und Moral Courage und DIAF-S sind Tab. 4 zu entnehmen.

$\operatorname{TiB}(r=0,25, p<0,05), \operatorname{VMP}(r=0,29, p<0,01)$ und der Gesamtwert des DIAF-S $(r=0,30, p<0,01)$ korrelierten signifikant positiv mit der durch die Führungskraft eingeschätzten Leistung der Mitarbeitenden. Für SB $(r=0,11$, $p=0,320)$ und AIV $(r=0,18, p=0,102)$ fanden sich keine signifikanten Zusammenhänge.

Promotive und prohibitive Voice zeigten ähnliche $\mathrm{Zu}$ sammenhänge zu authentischer Führung: SB korrelierte jeweils am höchsten (promotive Voice: $r=0,44, p<0,006$; prohibitive Voice: $r=0,50, p<0,006)$ mit beiden VoiceArten. Promotive und prohibitive Voice wiesen mit TiB $(0,32 \leq r \leq 0,36, p \leq 0,006)$ und $\operatorname{AIV}(0,28 \leq r=0,29, p \leq$ $0,01)$ kleine bis moderate Korrelationen auf. Zum Gesamtwert des DIAF-S ergaben sich für beide Voice-Arten moderate Korrelationen $(0,44 \leq r \leq 0,47 p<0,006)$. Für VMP und promotive $(r=0,15, p=0,172)$ sowie prohibitive Voice $(r=0,15, p=0,166)$ fanden sich keine Zusammenhänge.

$\mathrm{Zu}$ Moral Courage traten positive Zusammenhänge zwischen SB, TIB, AIV und dem Gesamtwert auf $(0,29 \leq r \leq$ $0,36, p \leq 0,01)$. Es ließ sich kein Zusammenhang zwischen Moral Courage und VMP feststellen $(r=0,09, p=0,413)$ (Tab. 4).

\subsection{Zusammenhänge zwischen Selbst- und Fremdeinschätzung}

Die deskriptiven Statistiken sowie Interkorrelationen der selbst- und fremdeingeschätzten authentischen Führung sind Tab. 5 zu entnehmen. 
Tab. 5 Deskriptive Statistiken, Reliabilitäten und Interkorrelationen der Selbst- und Fremdeinschätzung

\begin{tabular}{|c|c|c|c|c|c|c|c|c|c|c|c|c|c|c|c|}
\hline & $n$ & Min & Max & $M$ & $S D$ & 1 & 2 & 3 & 4 & 5 & 6 & 7 & 8 & 9 & 10 \\
\hline $\begin{array}{l}\text { 1. DIAF-S } \\
\text { SB }\end{array}$ & 42 & 2,75 & 4,75 & 3,99 & 0,47 & $(0,51)$ & & & & & & & & & \\
\hline $\begin{array}{l}\text { 2. DIAF-S } \\
\text { TiB }\end{array}$ & 42 & 2,75 & 5,00 & 4,07 & 0,62 & $0,54 * *$ & $(0,79)$ & & & & & & & & \\
\hline $\begin{array}{l}\text { 3. DIAF-S } \\
\text { VMP }\end{array}$ & 42 & 3,00 & 5,00 & 4,26 & 0,52 & 0,25 & 0,26 & $(0,72)$ & & & & & & & \\
\hline $\begin{array}{l}\text { 4. DIAF-S } \\
\text { AIV }\end{array}$ & 42 & 3,25 & 5,00 & 4,23 & 0,50 & $0,63 * *$ & $0,34^{*}$ & 0,23 & $(0,71)$ & & & & & & \\
\hline $\begin{array}{l}\text { 5. DIAF-S } \\
\text { Gesamt }\end{array}$ & 42 & 3,19 & 4,88 & 4,14 & 0,41 & $0,82 * *$ & $0,76^{* *}$ & $0,56 * *$ & $0,76^{* *}$ & $(0,86)$ & & & & & \\
\hline $\begin{array}{l}\text { 6. DIAF-F } \\
\text { SB }\end{array}$ & 259 & 1,00 & 5,00 & 3,47 & 0,93 & $\mathbf{0 , 3 5} *$ & $0,51 * *$ & 0,05 & 0,22 & $0,41 *$ & $(0,84)$ & & & & \\
\hline $\begin{array}{l}\text { 7. DIAF-F } \\
\text { TiB }\end{array}$ & 259 & 1,00 & 5,00 & 3,74 & 0,94 & $0,51 * *$ & $0,62^{* * *}$ & 0,27 & $0,33^{*}$ & $0,60 * *$ & $0,87 * *$ & $(0,82)$ & & & \\
\hline $\begin{array}{l}\text { 8. DIAF-F } \\
\text { VMP }\end{array}$ & 258 & 1,50 & 5,00 & 3,95 & 0,73 & 0,29 & $0,41 *$ & 0,09 & 0,10 & $0,32 *$ & $0,81 * *$ & $0,77 * *$ & $(0,81)$ & & \\
\hline $\begin{array}{l}\text { 9. DIAF-F } \\
\text { AIV }\end{array}$ & 258 & 1,25 & 5,00 & 3,66 & 0,95 & $0,31 *$ & $0,44 * *$ & 0,01 & 0,16 & $0,33 *$ & $0,91 * *$ & $0,81 * *$ & $0,77 * *$ & $(0,87)$ & \\
\hline $\begin{array}{l}\text { 10. DIAF-F } \\
\text { Gesamt }\end{array}$ & 259 & 1,38 & 5,00 & 3,70 & 0,79 & $0,40 *$ & $0,54 * *$ & 0,11 & 0,22 & $0,46^{* * *}$ & $0,97 * *$ & $0,93 * *$ & $0,89 * *$ & $0,94 * *$ & $(0,94)$ \\
\hline
\end{tabular}

Partialkorrelationen (Kontrollvariablen: Alter, Tätigkeitsdauer). Fett hervorgehobene Koeffizienten kennzeichnen die Korrelationen der korrespondierenden Komponenten des DIAF-S und DIAF-F. In den Klammern der Diagonalen sind die internen Konsistenzen (Cronbachs Alpha) der (Sub-)Skalen angegeben. Es ergeben sich durch einzelne fehlende Werte unterschiedliche Stichprobengrößen

$D I A F-S$ Deutsches Inventar Authentischer Führung als Selbsteinschätzungsform, DIAF-F Deutsches Inventar Authentischer Führung als Fremdeinschätzungsform, $S B$ Selbstbewusstheit, TiB Transparenz in Beziehungen zu anderen, VMP verinnerlichte moralische Perspektive, $A I V$ ausgewogene Informationsverarbeitung

*Die Korrelation ist auf dem Niveau von 0,05 signifikant (2-seitig); **Die Korrelation ist auf dem Niveau von 0,005 signifikant (2-seitig, Bonferroni-korrigiertes Niveau)

Für die Interrater-Übereinstimmung der Fremdeinschätzung des Führungsstils ergaben sich für verschiedene Verteilungsannahmen (stetig und leicht schief) für alle Komponenten und die Gesamtskala Werte, die nach LeBrenton und Senter (2008) auf eine moderate bis hohe Übereinstimmung innerhalb der Teams hinweisen. Für die Annahme einer stetigen Gleichverteilung wies die Komponente AIV den niedrigsten Wert, die Komponente VMP den höchsten Wert in der Übereinstimmung auf $\left(0,68 \leq r_{w g} \leq 0,81\right)$. Für die Annahme einer leicht schiefen Verteilung erreichte die Komponente AIV den niedrigsten Wert, die Komponente VMP den höchsten $\left(0,52 \leq r_{w g} \leq 0,71\right)$.

Aufgrund moderater bis hoher Korrelationen zwischen verschiedenen Komponenten und der Gesamtskala selbsteingeschätzter authentischer Führung mit Alter $(0,38 \leq r \leq 0,55, p<0,05)$ und mit Tätigkeitsdauer $(0,44 \leq r \leq 0,57, p<0,05)$ wurden nachfolgend Partialkorrelationen gerechnet.

Zwischen der Fremd- und Selbsteinschätzung fanden sich für die Gesamtskala $(r=0,46, p<0,005)$ und für SB $(r=0,35, p<0,05)$ und TiB $(r=0,62, p<0,005)$ moderate bis hohe Zusammenhänge. Bei AIV korrelierten Selbst- und Fremdeinschätzung nicht miteinander $(r=0,16, p=0,325)$, auch bei der Komponente VMP bestand keine Korrelation $(r=0,09, p=0,565)$.

\section{Diskussion}

Der Beitrag umfasst die Prüfung und Validierung der ersten deutschen Selbsteinschätzungsform des Inventars zur Erfassung authentischer Führung - DIAF-S.

Geprüft wurden die Güte und die faktorielle Binnenstruktur. Zur Beurteilung der Kriteriumsvalidität wurden organisationale Außenkriterien - Leistung, Voice-Verhalten und Moral Courage - herangezogen und der Zusammenhang zwischen selbst- und fremdeingeschätzter authentischer Führung betrachtet. Das DIAF-S wurde mittels Selbsteinschätzungen von $N=91$ Führungskräften und Fremdeinschätzungen von $N=259$ Mitarbeitenden geprüft.

Zwischen authentischer Führung und Alter sowie Tätigkeitsdauer traten signifikante Zusammenhänge auf. Ältere und länger tätige Führungskräfte schätzten sich auf allen Ebenen authentisch führender ein als jüngere bzw. als Führungskräfte, die kürzer tätig waren. Eine Erklärung könnte darin bestehen, dass ein positives Selbstbild und die Wahrnehmung des eigenen Führungsverhaltens über die Zeit hinweg durch passende Situationen bestätigt und verstärkt werden.

Die Itemschwierigkeiten sind als leicht einzuordnen. Entsprechend schätzten sich die Führungskräfte im Durschnitt auf allen Komponenten hoch ein, was durch den 
Vergleich mit der Fremdeinschätzungsform (DIAF-F, Franke-Bartholdt et al. 2018) mit mittleren Itemschwierigkeiten, zusätzlich verdeutlicht wird. Bei einem Selbsteinschätzungsverfahren wie dem DIAF-S sind erhöhte Mittelwerte zu erwarten, da insbesondere Führungskräfte einem positiven Bias gegenüber dem eigenen Verhalten unterliegen können (Yammarino und Atwater 1997). Die Items repräsentieren erstrebenswertes Verhalten, sodass sozial erwünschtes Antwortverhalten wahrscheinlicher wird. Die Differenzierung zwischen Personen mit hoher und niedriger Merkmalsausprägung wird demnach erschwert. Insbesondere die Komponente VMP, welche das Ausrichten der Führungskraft an hohen moralischen Standards erfasst, wies den höchsten Mittelwert und die geringste Trennschärfe auf. Umso wichtiger ist, die beiden Formen zur Selbst- und Fremdeinschätzung ergänzend einzusetzen, um beide Perspektiven zu erfassen. Die Itemtrennschärfen lagen für den Großteil der Items im angestrebten mittleren Bereich, wobei zwei Items der Komponente SB mit einer Trennschärfe $r_{\mathrm{it}}<0,30$ als kritisch zu betrachten sind. Gegenüber den hervorragenden Trennschärfen des DIAF-F fallen die Trennschärfen des DIAF-S allerdings deutlich geringer aus, was auf die homogene Stichprobe und eine damit verbundene Varianzeinschränkung zurückzuführen sein kann.

Die psychometrischen Eigenschaften des DIAF-S sind überwiegend als befriedigend bis gut zu bewerten. Die Reliabilitäten der Komponenten TiB, AIV, VMP und der Gesamtskala lagen im befriedigenden bis guten Bereich, während die Reliabilität von $\alpha=0,55$ für die Komponente SB nicht als zufriedenstellend angesehen werden kann. Daher sollten die Items der Komponente SB überarbeitet werden. Einen Ansatz liefert das Ergebnis der EFA, bei welcher sich die Items der Komponenten SB und TiB gemeinsam auf Faktor 1 wiederfanden, während Item 1 der Komponente SB gemeinsam mit den Items der Komponente AIV auf Faktor 2 lud. Bei einer explorativen Betrachtung der Reliabilität für die Faktorenlösung der EFA ergaben sich befriedigende interne Konsistenzen: Für Faktor 1 mit drei Items der Komponente SB und vier Items der Komponente TiB ein Cronbachs Alpha von 0,74, für Faktor 2 mit Item 1 der Komponente SB und vier Items der Komponente AIV ein Cronbachs Alpha von 0,66 und für Faktor 3 mit den Items der Komponente VMP ein Wert von 0,65. Durch die Hinzunahme von Item $1 \mathrm{zu}$ der Komponente AIV fällt die Reliabilität von 0,69 auf 0,66 , weswegen eine Überarbeitung von Item 1 erfolgen sollte.

Bei der Prüfung der Binnenstruktur des DIAF-S und DIAF-F per CFA wurden analog zum Vorgehen von Franke-Bartholdt et al. (2018) verschiedene Modelle geprüft: Modell 1 mit vier interkorrelierten Faktoren, Modell 2 mit vier Faktoren erster und einem Faktor zweiter Ordnung,
Modell 3 mit zwei interkorrelierten Faktoren und Modell 4 mit einem Faktor.

Für das DIAF-S erreichten das Modell 1 mit vier interkorrelierten Faktoren und das Modell 2 mit vier Faktoren erster und einem Faktor zweiter Ordnung gleiche Kennwerte in den Fit-Indizes und unterschieden sich nicht signifikant voneinander. Beide Modelle bildeten die Daten im Vergleich zu den Alternativmodellen signifikant besser ab, wiesen allerdings einen eingeschränkten Gesamt-Fit auf. Aus Sparsamkeitsgründen sollte auf das weniger komplexe Modell 1 mit vier interkorrelierten Faktoren SB, TiB, AIV und VMP zurückgegriffen werden.

Für das DIAF-F wies das Modell mit vier interkorrelierten Faktoren einen zufriedenstellenden Gesamt-Fit auf und war den drei Alternativmodellen signifikant überlegen. Somit konnte die von Franke-Bartholdt et al. (2018) gefundene vierfaktorielle Struktur für die Fremdeinschätzungsform authentischer Führung bestätigt werden.

Zusammenfassend erklärte ein Modell mit den interkorrelierten Faktoren SB, TiB, AIV und VMP beide Formen zur Beurteilung authentischer Führung im Vergleich zu den Alternativmodellen am besten. Die vierfaktorielle Struktur entspricht der von Walumbwa et al. (2008) postulierten, welche mehrfach empirisch nachgewiesen werden konnte (Franke-Bartholdt et al. 2018; Neider und Schriesheim 2011). Entsprechend ist ein Vergleich der Selbst- und Fremdeinschätzungsform auf Komponentenebene möglich.

Für das DIAF-S konnte allerdings kein zufriedenstellender Gesamt-Fit erreicht werden. Die Ergebnisse der CFA sollten aufgrund der geringen Stichprobengröße von $n=80$ mit Vorsicht interpretiert werden, da bei kleinen Stichproben häufiger Schätzprobleme auftreten (Bühner 2010) und die vorliegende Anzahl an ProbandInnen unter den Empfehlungen der erforderlichen Größe zur Berechnung von CFA liegt (Schermelleh-Engel et al. 2003).

Die unzureichende Passung eines Modells mit vier interkorrelierten Faktoren für das DIAF-S gibt Anlass, die Struktur authentischer Führung in der Selbsteinschätzung weiter zu erforschen. Einen Ansatz dazu liefert das Ergebnis der EFA, bei der sich eine dreifaktorielle Lösung ergab. Die Items der Komponenten SB und TiB fanden sich auf einem gemeinsamen Faktor wieder, lediglich Item 1 der Komponente SB lud mit den Items der Komponente AIV auf Faktor 2. Wenn man das Item betrachtet („Ich erbitte Rückmeldung, um meinen Umgang mit anderen zu verbessern.") wird deutlich, dass es inhaltlich um das Einholen von Informationen geht, was eine für AIV charakteristische Prozesskomponente nahelegt. Auf Grund der geringen Trennschärfe $\left(r_{\mathrm{it}-\mathrm{K}}=0,22\right)$ und der hohen Hauptladung auf Faktor 2 sollte das Item sprachlich überarbeitet oder entfernt werden. Die mit explorativen Analysen gefundene Faktorenstruktur entspricht nicht der von Walumbwa et al. (2008) postulierten Struktur, lässt sich aber mit einem alternativen 
Modell authentischer Führung nachvollziehbar erklären: In der Theory of Embodied Authentic Leadership (Ladkin und Taylor 2010) findet sich der Verhaltensaspekt der Selbstgesinnung, der die Komponenten SB und TiB beschreibt. Selbstgesinnung bedeutet, ein tiefgehendes Bewusstsein für das eigene Gefühlsleben zu haben und entscheiden zu können, ob Emotionen zum Ausdruck gebracht werden. Wichtig ist dabei, dass Führungskräfte auch Schwächen zeigen können und sich Mitarbeitende über deren offenen Umgang damit mit der Führungskraft identifizieren. Dies fördert das Vertrauen und tiefere Beziehungen. Somit kann die Komponente SB als vorausgehende Bedingung angenommen werden, die TiB fördert. Die zweite Verhaltenskomponente $B e$ züge herstellen nach Ladkin und Taylor (2010) kann als Pendant zur Komponente AIV gesehen werden. Es geht um die Wichtigkeit des sozialen Austausches, um Entscheidungen zum Wohl der Organisation zu treffen. Hierbei können auch Meinungen geäußert werden, die nicht in Übereinstimmung mit denen der Führungskraft stehen. Diese muss sich infolgedessen an die kontextuellen Anforderungen anpassen und Informationen von den Mitarbeitenden einholen. Zuletzt beinhaltet die Verhaltenskomponente der Führungsentscheidungen, dass die Führungskraft die Identität der Gruppe spiegelt und den Rahmen für Entwicklungsprozesse schafft, was durch geteilte moralische Werte gefördert wird. Entsprechend kann dies der DIAF-S Komponente der VMP zugeordnet werden. Ein weiteres dreifaktorielles Modell authentischer Führung stellt Whitehead (2009) vor. Eine authentische Führungskraft ist ,(1) (...) self-aware, humble, always seeking improvement, aware of those being led and looks out for the welfare of others; (2) fosters high degrees of trust by building an ethical and moral framework; and (3) is committed to organizational success within the construct of social values" (Whitehead 2009, S. 850). Die Eigenschaften unter (1) bilden damit die Komponenten SB und $\mathrm{TiB} \mathrm{ab}$, unter (2) werden Aspekte der Komponente VMP dargestellt und (3) deckt sich im weiteren Sinne mit der Komponente AIV.

Die erwarteten moderaten Zusammenhänge zwischen den Außenkriterien Arbeitsleistung, Voice und Moral Courage konnten für $\mathrm{SB}$, TiB und $\mathrm{AIV}$ und den Gesamtwert bestätigt werden und sind als vielversprechend $\mathrm{zu}$ werten. So fanden sich ähnliche hohe Zusammenhänge für die Kriteriumsvalidität wie beim DIAF-F. Hypothesenkonträr fielen die Zusammenhänge zwischen VMP und promotive und prohibitive Voice und Moral Courage aus. Hier ergaben sich kleine, nicht signifikante Korrelationen. Die befragten Führungskräfte schätzten sich sehr homogen auf allen Komponenten, insbesondere aber bei VMP ein, sodass hier mit einer Varianzeinschränkung und damit Korrelationsminderung zu rechnen ist. Zusätzlich spiegeln die Items zur verinnerlichten moralischen Perspektive innere Vorgänge wider und kein direkt beobachtbares Verhalten. Damit die- ser Aspekt authentischer Führung mit sichtbarem, aktivem Verhalten wie Voice und Moral Courage zusammenhängen kann, muss das Verhalten auch wahrnehmbar sein.

Bei der Betrachtung der Übereinstimmung zwischen Selbst- und Fremdeinschätzung wurden moderate bis hohe Werte für die Übereinstimmung der Mitarbeitenden innerhalb eines Teams erreicht. Damit kann die Bildung des Mittelwerts als zulässig erklärt werden (LeBreton und Senter 2008). Allerdings ist zu beachten, dass die Varianz in der Übereinstimmung recht hoch ausfiel. Die Einschätzung des Führungsstils kann durchaus auch innerhalb eines Teams variieren, da eine Führungskraft auf Grund von Unterschieden im Leader-Member-Exchange zu verschiedenen Mitarbeitenden eine unterschiedliche Beziehungsqualität haben kann. Dies kann zu unterschiedlichen Beurteilungsmustern des Führungsstils führen (Estel et al. 2019). Für die Komponenten SB, TiB und den Gesamtwert fanden sich moderate bis hohe Zusammenhänge zwischen Selbst- und Fremdeinschätzung. Der größte Zusammenhang bestand für die Komponente TiB: Diese bildet den relationalen Aspekt zwischen Mitarbeitenden und Führungskräften ab und kann daher von beiden Seiten gleichermaßen gut über gezeigtes Verhalten eingeschätzt werden. Die Schwierigkeit der Einschätzung nicht sichtbaren Verhaltens einer verinnerlichten moralischen Perspektive zeigte sich auch durch den nicht gefundenen Zusammenhang zwischen Fremdund Selbsteinschätzung. Metanalytisch wurde für ethische Führung, die große Überschneidungen mit authentischer Führung aufweist, die größte Effektstärke gegenüber transformationaler oder dienender Führung für den Unterschied zwischen Selbst- und Fremdeinschätzung gefunden (Lee und Carpenter 2018). Es ist anzunehmen, dass gerade bei Inhalten zu moralischem Verhalten eine große Diskrepanz zwischen Selbst- und Fremdeinschätzung auftritt.

\section{Limitationen und Empfehlungen}

Die Prüfung und Validierung des DIAF-S wurde durch ein konkurrentes Design realisiert. Damit können keine Aussagen zur prädiktiven Validität des DIAF-S getroffen werden. Dies ließe sich zukünftig durch längsschnittliche Untersuchungen lösen. Die Führungskräfte schätzen sich bezüglich authentischer Führung sehr homogen ein, was die gefundenen Zusammenhänge möglicherweise eingeschränkt abbildet. Zudem war auch die Stichprobe sehr homogen, da alle befragten Führungskräfte im Gesundheitssektor arbeiteten. Die Testung des DIAF-S an einer größeren, heterogenen Stichprobe von Führungskräften ist daher zu empfehlen, insbesondere in Hinblick auf die Überprüfung der faktoriellen Validität. Vor allem Branchen, in denen moralische Standards eine bedeutende Rolle zukommt, sollten einbezogen werden. Um umfassende Aussagen zur Kriteriums- 
validität treffen zu können, wären Befunde zu affektiven Kriterien der Mitarbeitenden (Wohlbefinden, Arbeitsengagement), aber auch zu relationalen (Leader-Member-Exchange), wie es im DIAF-F umgesetzt wurde, aufschlussreich. Die Überprüfung der faktoriellen Validität mittels CFA liefert aktuell keine zufriedenstellenden Modell-Fits. Das die theoretische Konzipierung authentischer Führung noch nicht abgeschlossen ist, zeigt sich auch durch die in der EFA gefundenen dreifaktoriellen Struktur. Ein Modell mit drei interkorrelierten Faktoren sollte daher in weiteren Studien gezielter geprüft werden. Es bedarf weiterer Forschung, um das Konstrukt der authentischen Führung zu schärfen und die einzelnen Komponenten trennschärfer voneinander abzugrenzen bzw. zu einer für die Selbstbeurteilung passenderen Struktur zu kommen.

\section{Transfer}

Mit dem DIAF-S liegt nun neben der Fremdeinschätzungsform, dem DIAF-F, ein weitgehend reliables und valides Instrument zur Selbstbeurteilung authentischen Führungsverhaltens vor. Die Ergebnisse dieser Studie weisen auf überwiegend gute psychometrische Eigenschaften hin und zeigen bezüglich organisationaler Außenkriterien vielversprechende Ergebnisse. Zu berücksichtigen ist die niedrige interne Konsistenz der Komponente SB, weswegen bei einer Einzelnutzung des DIAF-S eine alternative Struktur authentischer Führung empfohlen wird. Diese basiert auf drei Faktoren, die die Selbsteinschätzungsform anhand theoretischer Alternativmodelle erklären kann und bei deren Anwendung sich interne Konsistenzen im befriedigenden Bereich $(0,65 \leq \alpha \leq 0,74)$ ergaben.

Für den Abgleich zwischen Selbst- und Fremdeinschätzung kann auf das vierfaktorielle Modell mit den Komponenten SB, TiB, AIV und VMP zurückgegriffen werden und somit eine gemeinsame Nutzung des DIAF-S mit dem DIAF-F empfohlen werden. Mit Hilfe dieser beiden Formen des DIAF lassen sich multiperspektivisch Aussagen zum authentischen Führungsstil ableiten. Darin besteht ein immenser Vorteil gegenüber der alleinigen Betrachtung des Führungsverhaltens aus Selbst- oder Fremdperspektive, da gemeinsam Erwartungen an den Führungsstil eruiert und Verbesserungsmaßnahmen bei auftretenden Diskrepanzen eingeleitet werden können (Franke-Bartholdt et al. 2018).

Für das Führungskräfte-Coaching stellt das DIAF-S ein wichtiges Instrument dar, da mit den verhaltensnahen Items das Rollenverständnis und die Vorbildfunktion, insbesondere zum Umgang mit moralischen Sachverhalten, reflektiert werden können. Gerade in diesem Bereich weisen die Befunde dieser Studie auf eine große Diskrepanz zwischen selbst- und fremdwahrgenommen Verhalten hin.
Entsprechend kann der Abgleich zwischen den von den Mitarbeitenden wahrgenommenem Führungsverhalten und der Selbsteinschätzung der Führungskraft Anstoß geben, um Abweichungen zu ermitteln. Auf Basis dieser Abweichungen können Führungskräfte einen sicheren Umgang mit möglichen Unzulänglichkeiten als Führungskraft lernen und sich weiterentwickeln. Bei der Beurteilung der Selbsteinschätzung sollte auch der Kontext beachtet werden: Wenn es im Rahmen eines Führungskräfte-Coachings gezielt darum geht, den eigenen Führungsstil zu reflektieren und diesen weiter zu entwickeln, antworten Führungskräfte ehrlicher und gestehen Unzulänglichkeiten eher ein als in anderen Situationen (Lee und Carpenter 2018). Im Kontext der Standortbestimmung, wenn beispielsweise auf Basis des Führungsstils personelle Entscheidungen getroffen werden, wird sozial erwünschtes Antwortverhalten sehr wahrscheinlich. Aus diesem Grund sollten gerade dann Fremdbeurteilungen eingeholt werden, um diesen Effekt abzuschwächen.

Weiterhin kann mit Hilfe des DIAF das nomologische Netzwerk authentischer Führung erforscht und erweitert werden und Erkenntnisse und Wissen über eines der bedeutendsten Merkmale im organisationalen Kontext zu gewinnen: Sich selbst treu zu sein und damit eine Vertrauenskultur zu schaffen, die einen offenen Diskurs fördert.

Funding Open Access funding provided by Projekt DEAL.

Open Access Dieser Artikel wird unter der Creative Commons Namensnennung - Weitergabe unter gleichen Bedingungen 4.0 International Lizenz veröffentlicht, welche die Nutzung, Vervielfältigung, Bearbeitung, Verbreitung und Wiedergabe in jeglichem Medium und Format erlaubt, sofern Sie den/die ursprünglichen Autor(en) und die Quelle ordnungsgemäß nennen, einen Link zur Creative Commons Lizenz beifügen und angeben, ob Änderungen vorgenommen wurden. Wenn Sie den Artikel oder Teile daraus remixen, verändern oder anderweitig direkt darauf aufbauen, dürfen Sie Ihre Beiträge nur unter derselben Lizenz wie das Original verbreiten.

Die in diesem Artikel enthaltenen Bilder und sonstiges Drittmaterial unterliegen ebenfalls der genannten Creative Commons Lizenz, sofern sich aus der Abbildungslegende nichts anderes ergibt. Sofern das betreffende Material nicht unter der genannten Creative Commons Lizenz steht und die betreffende Handlung nicht nach gesetzlichen Vorschriften erlaubt ist, ist für die oben aufgeführten Weiterverwendungen des Materials die Einwilligung des jeweiligen Rechteinhabers einzuholen.

Weitere Details zur Lizenz entnehmen Sie bitte der Lizenzinformation auf http://creativecommons.org/licenses/by-sa/4.0/deed.de.

\section{Literatur}

Arbuckle, J.L. (2014). Amos (Version 26.0). Chicago, IL: IBM SPPS. Computer Program

Banks, G.C., McCauley, K.D., Gardner, W.L., \& Guler, C.E. (2016). A meta-analytic review of authentic and transformational leadership: a test for redundancy. The Leadership Quarterly, 27(4), 634-652.

Brown, M.E., \& Mitchell, M.S. (2010). Ethical and unethical leadership: exploring new avenues for future research. Business Ethics Quarterly, 20, 583-616. 
Brown, M.E., Treviño, L. K., \& Harrison, D. A. (2005). Ethical leadership: a social learning perspective for construct development and testing. Organizational Behavior and Human Decision Processes, 97, 117-134.

Bühner, M. (2010). Einführung in die Test- und Fragebogenkonstruktion (3. Aufl.). München: Pearson-Education.

Estel, V., Schulte, E. M., Spurk, D., \& Kauffeld, S. (2019). LMX differentiation is good for some and bad for others: a multilevel analysis of effects of LMX differentiation in innovation teams. Cogent Psychology, 6(1), 1614306.

Franke-Bartholdt, L., Frömmer, D., Wegge, J., \& Strobel, A. (2018). Authentische Führung: Entwicklung und Validierung einer modifizierten deutschen Fassung des Authentic Leadership Inventory von Neider und Schriesheim. Zeitschrift für Arbeits- und Organisationspsychologie A\&O, 62(3), 142-160.

Hannah, S. T., \& Avolio, B. J. (2010). Moral potency: building the capacity for character-based leadership. Consulting Psychology Journal: Practice and Research, 62(4), 2919-2310.

Hannah, S. T., Avolio, B. J., \& Walumbwa, F. O. (2011). Relationships between authentic leadership, moral courage, and ethical and prosocial behaviors. Business Ethics Quarterly, 21(4), 555-578.

Hoch, J. E., Bommer, W. H., Dulebohn, J.H., \& Wu, D. (2018). Do ethical, authentic, and servant leadership explain variance above and beyond transformational leadership? A meta-analysis. Journal of Management, 44(2), 501-529.

Horn, J.L. (1965). A rationale and test for the number of factors in factor analysis. Psychometrika, 30(2), 179-185.

Hu, L., \& Bentler, P. M. (1999). Cutoff criteria for fit indexes in covariance structure analysis: conventional criteria versus new alternatives. Strucutural Equation Modelling, 6, 1-29.

IBM Corp. (2017). IBM SPSS Statistics for Macintosh, Version 25.0. Armonk, NY: IBM Corp.

Kernis, M.H. (2003). Toward a conceptualization of optimal self-esteem. Psychological Inquiry, 14, 1-26.

Ladkin, D., \& Taylor, S.S. (2010). Enacting the 'true self': towards a theory of embodied authentic leadership. The Leadership Quarterly, 21(1), 64-74.

LeBreton, J. M., \& Senter, J.L. (2008). Answers to 20 questions about interrater reliability and interrater agreement. Organizational research methods, 11(4), 815-852.

Lee, A., \& Carpenter, N.C. (2018). Seeing eye to eye: a meta-analysis of self-other agreement of leadership. The Leadership quarterly, 29(2), 253-275.

Leroy, H., Anseel, F., Gardner, W.L., \& Sels, L. (2015). Authentic leadership, authentic followership, basic need satisfaction, and work role performance: a cross-level study. Journal of management, 41(6), 1677-1697.

Liang, J., Farh, C. I. C., \& Farh, J.-L. (2012). Psychological antecedents of promotive and prohibitive voice: a two-wave examination. Academy of Management Journal, 55(1), 71-92.

Luthans, F., \& Avolio, B. J. (2003). Authentic leadership development. In K.S. Cameron, J.E. Dutton \& R.E. Quinn (Hrsg.), Positive organizational scholarship: foundations of a new discipline (S. 241-261). San Francisco: Barrett-Koehler.

Neider, L.L., \& Schriesheim, C. A. (2011). The authentic leadership inventory (ALI): development and empirical tests. The Leadership Quarterly, 22, 1146-1164.

O'Connor, B.P. (2000). SPSS and SAS programs for determining the number of components using parallel analysis and Velicer's MAP test. Behavior Research Methods, Instrumentation, and Computers, 32, 396-402.

Robinson, S.L. (1996). Trust and breach of the psychological contract. Administrative Science Quarterly, 41, 574-599.

Rose, A. (2015). Übersetzung und Prüfung der Instrumente Moral Potency Questionnaire, Silence Motives und Promotive and Prohibitive Voice. Unveröffentlichte Studienabschlussarbeit, Technische Universität Chemnitz.
Schermelleh-Engel, K., Moosbrugger, H., \& Müller, H. (2003). Evaluating the fit of structural equation models: tests of significance and descriptive goodness-of-fit measures. Methods of psychological research online, 8(2), 23-74.

Steiger, J.H. (1990). Structural model evaluation and modification: an interval estimation approach. Multivariate behavioral research, 25(2), 173-180.

Tepper, B. J. (2000). Consequences of abusive supervision. Academy of Management Journal, 43, 178-190.

Treviño, L.K., den Nieuwenboer, N. A., \& Kish-Gephart, J. J. (2014). (Un)Ethical behavior in organizations. Annual Review of Psychology, 65, 635-660.

Walumbwa, F. O., Avolio, B. J., Gardner, W.L., Wernsing, T. S., \& Peterson, S. J. (2008). Authentic leadership: development and validation of a theory-based measure. Journal of Management, 34, 89-126.

West, S. G., Finch, J.F., \& Curran, P.J. (1995). Structural equation models with nonnormal variables: problems and remedies. In R.H. Hoyle (Hrsg.), Structural equation modeling: concepts, issues, and applications (S. 56-75). Thousand Oaks: SAGE.

Whitehead, G. (2009). Adolescent leadership development: building a case for an authenticity framework. Educational Management Administration \& Leadership, 37(6), 847-872.

Wong, C. A., \& Cummings, G. G. (2009). The influence of authentic leadership behaviors on trust and work outcomes of health care staff. Journal of Leadership Studies, 3(2), 6-23.

Yammarino, F. J., \& Atwater, L. E. (1997). Do managers see themselves as other see them? Implications of self-other rating agreement for human resources management. Organizational Dynamics, 25(4), $35-44$.

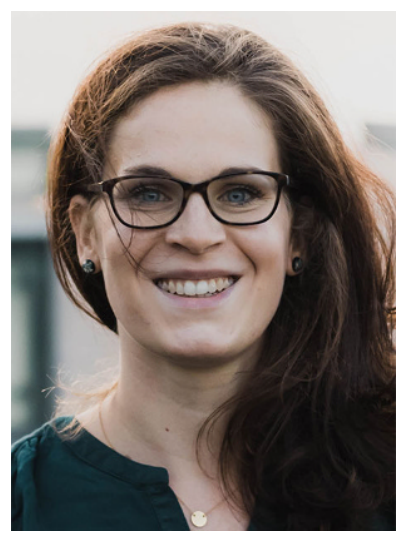

Magdalena Reineboth Dipl.Psych. Magdalena Reineboth ist seit 2016 wissenschaftliche Mitarbeiterin an der Professur für Persönlichkeitspsychologie und Diagnostik an der Technischen Universität Chemnitz. In ihrer Promotion beschäftigt sie sich mit Verhalten in moralisch relevanten Situationen am Arbeitsplatz und der Entwicklung eines Testverfahrens, um dieses objektiv und valide zu erfassen. 


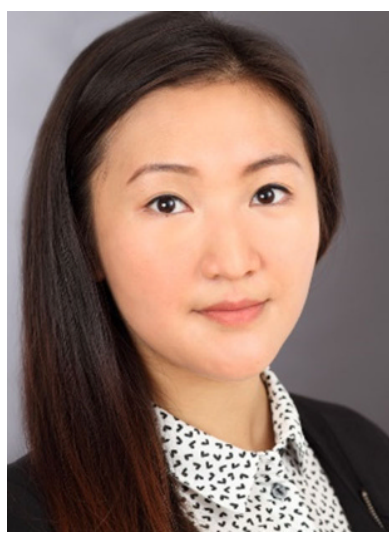

Nensy Le Thu Ha hat sich in ihrer Bachelorarbeit mit der Validierung der Selbsteinschätzungsform des Deutschen Inventars Authentischer Führung (DIAF-S) auseinandergesetzt. Aktuell absolviert sie ihr Masterstudium an der Universität Leipzig.

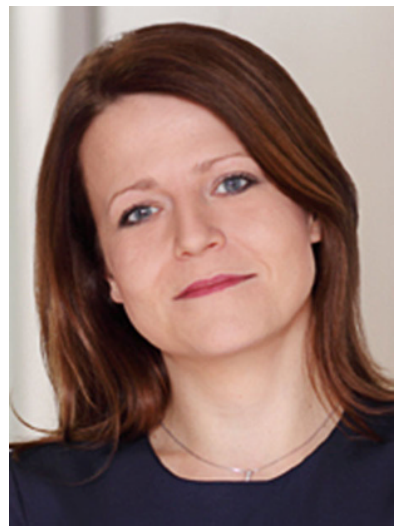

Luise Franke-Bartholdt Dipl.Psych. Luise Franke-Bartholdt ist als Agenturpsychologin in einem Berufspsychologischen Service der Bundesagentur für Arbeit tätig. Sie war von 2009 bis 2017 als wissenschaftliche Mitarbeiterin an der Professur für Persönlichkeitspsychologie und Diagnostik an der Technischen Universität Chemnitz beschäftigt und hat u. a. an der Entwicklung von Verfahren zur Erfassung von Authentischer Führung und moralisch motiviertem Verhalten mitgewirkt.

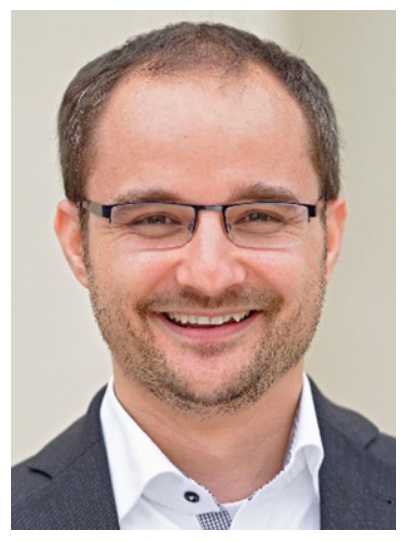

Dirk Frömmer Dipl.-Psych. Dirk Frömmer ist Partner und geschäftsführender Gesellschafter bei Management Innovation Dresden und dort als Berater und Trainer in den Feldern Organisations-, Personalund Teamentwicklung tätig. Des Weiteren ist er Doktorand an der Professur für Arbeits- und Organisationspsychologie an der Technischen Universität Dresden. In seiner Promotion beschäftigt er sich mit den Einflüssen authentischer Führung auf das Schweigen und VoiceVerhalten von Mitarbeiterinnen und Mitarbeitern bei moralischen Sachverhalten und Problemen.

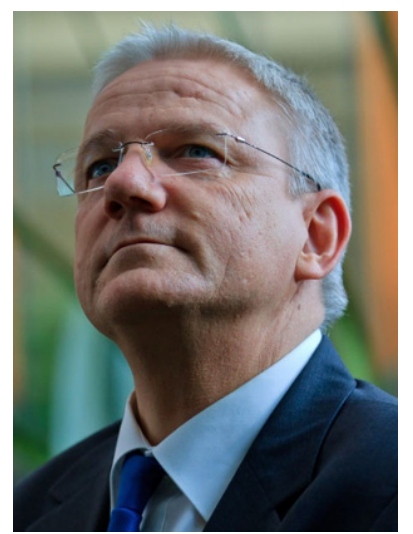

Jürgen Wegge Herr Dr. Jürgen Wegge ist seit 2007 Professor für Arbeits- und Organisationspsychologie an der TU Dresden (Promotion 1994 und Habilitation 2003 an der Universität Dortmund). Er war von 2010 bis 2012 Präsident der Fachgruppe Arbeits-, Organisations- und Wirtschaftspsychologie der DGPs. Seit Juni 2016 ist er Vorsitzender des Centrum für Demografie und Diversität (CDD) an der TU Dresden (https://tu-dresden.de/cdd). Seine Arbeitsschwerpunkte liegen im Bereich Arbeitsmotivation, Führung, demografischer Wandel, Arbeit und Gesundheit sowie Diversity in Organisationen. $\mathrm{Zu}$ diesen Themen hat er fünf Bücher, sieben special issues, 89 Zeitschriftenartikel und 108 Buchkapitel publiziert.

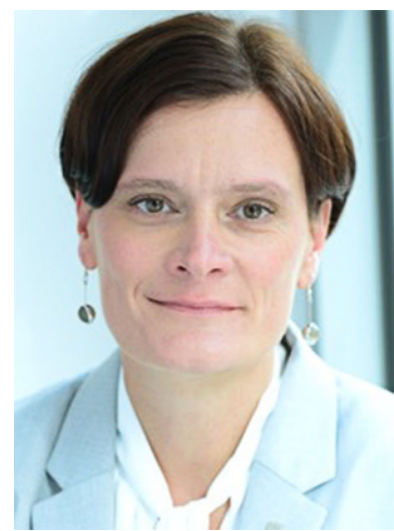

Anja Strobel Dr. Anja Strobel ist Universitätsprofessorin für Persönlichkeitspsychologie und Diagnostik an der Technischen Universität Chemnitz. Ihre Forschungsschwerpunkte sind Bedingungen und Konsequenzen (un-)moralischen Verhaltens von Mitarbeitenden in Organisationen, das Persönlichkeitsmerkmal kognitive Motivation sowie die Aussagekraft von Auswahlprozessen und die Kompetenz von Interviewenden. Ihre bisherigen Arbeiten mündeten in zahlreiche Buchbeiträge und Zeitschriftenartikel sowie Fragebogen.

Frau Strobel setzt sich unter anderem als Mitglied des wissenschaftlichen Beirats des Forums Assessment sowie als Trainerin im Rahmen der DIN 33430-Personenlizenzierung für eine hohe Qualität der Eignungsbeurteilung und enge Verzahnung von Forschung und Praxis ein. Den Transfer praxisrelevanten Wissens treibt sie über zahlreiche Projekte mit Industriepartnern sowie als Dozentin und Referentin in verschiedenen Kontexten voran. 